\title{
Significados de los procesos educativos para profesionales de la salud que se desempeñan en un centro de diálisis
}

\author{
Eugenia Karina Sanhueza-Inzunza, Carolina Alejandra Aparicio-Molina \\ Universidad Católica de la Santísima Concepción. Talcahuano. Chile
}

Como citar este artículo:

Sanhueza-Inzunza EK D, Aparicio Molina CA. Significados de los procesos educativos para profesionales de la salud que se desempeñan en un centro de diálisis.

Enferm Nefrol. 2020 Jul-Sep;23(3):252-258

\section{Resumen}

Objetivo: Cuando un paciente ingresa a diálisis se generan importantes cambios que afectan el estilo de vida de ellos y su familia. Para abordar este ingreso, los profesionales de la salud deben implementar procesos de enseñanza orientados a mejorar la calidad de vida de los pacientes, además de salvarla.

Objetivo: Debido a lo relevante de estos procesos educativos, el estudio que se presenta a continuación buscó comprender los significados que otorgan los profesionales de la salud a los procesos educativos que desarrollan en usuarios con insuficiencia renal crónica en un centro de diálisis.

Material y Método: La investigación de tipo cualitativa, de diseño fenomenológico e interpretativo. La información generada a través de entrevistas en profundidad a profesionales médicos y de enfermería, fue analizada por medio del método de análisis de contenidos.

Resultados: Los resultados obtenidos permitieron comprender significados asociados a los periodos de educación, métodos utilizados y principalmente los elementos que facilitaban y obstaculizaban estos procesos.

Conclusión: Se concluye que los profesionales de la salud trabajan en base a un método no formalizado, donde

\section{Correspondencia:}

Eugenia Sanhueza Inzunza

Email:esanhueza@magisteredu.ucsc.cl el diálogo y la experiencia o trayectoria resultan fundamentales para generar estos procesos de enseñanza.

PALABRAS CLAVE: educación profesional; educación en salud; diálisis.

•

Meaning of the educational processes for health professionals who work in a dialysis centre

\section{Abstract}

Introduction: The patient's admission to dialysis causes important changes that impact their lifestyle and family. To address this admission, health professionals must implement teaching processes aimed at improving the quality of life of patients, as well as save.

Objective: Due to the relevance of educational processes, the present study sought to understand the meanings that health professionals give to educational processes, which they implement in users with chronic kidney disease in a dialysis centre.

Material and Method: Qualitative research, phenomenological and interpretive design. The information generated through in-depth interviews with medical and nursing professionals was analysed using the content analysis method.

Results: The results obtained allow to understand meanings associated with periods of education, methods 
and mainly the elements that facilitated and hindered these processes.

Conclusion: It is concluded that health professionals work based on a non-formalized method, where dialogue and experience or trajectory are essential to generate these teaching processes.

KEY WORDS: professional education; health education; dialysis.

\section{Introducción}

Según datos de la Organización Mundial de la Salud las enfermedades no transmisibles (ENT) matan a $41 \mathrm{mi}-$ Ilones de personas cada año, lo que equivale al $71 \%$ de todas las muertes a nivel mundial ${ }^{1}$. Para abordar esta situación, cuando un usuario es diagnosticado con una enfermedad crónica, se enfrenta a una serie de información proveniente de los centros médicos, para mejorar su condición de salud, debiendo modificar muchas veces sus hábitos y estilo de vida. En el caso de la insuficiencia renal crónica terminal (IRCT), en el ingreso de los usuarios a centros de diálisis, son inmediatamente sometidos a un intenso repertorio de medicamentos, procedimientos y sugerencias de modificaciones tanto en su dieta, como estilos de vida que crean en ellos una infinidad de inquietudes y nuevas condiciones ${ }^{2}$. Los procesos de educación ofrecidos en este contexto, no sólo permiten que los usuarios aumenten sus conocimientos acerca de la enfermedad, sino que también mejoren su calidad de vida, y con ello la adherencia al tratamiento ${ }^{3}$. Estos procesos son generados por profesionales de la salud médica y de enfermería siendo su rol de vital importancia.

Este estudio se justifica en tanto, los usuarios se enfrentan a la realidad de ingresar a un tratamiento crónico que deben aprender a conocer y aceptar. Por ello se vuelve fundamental conocer los procesos de educación en salud del usuario, siendo importante comprender los significados que le otorgan los profesionales de la salud a los procesos de educativos de usuarios en diálisis.

Entendemos por educación en salud del paciente cuando éste es capaz de gestionar su propia enfermedad, contribuyendo a un aumento considerable de su estado de salud y de su calidad de vida ${ }^{4}$, donde su motivación por aprender no es aprobar una materia, sino comprender más acerca de su enfermedad ${ }^{5}$. Cada paciente es distinto, con una realidad y entorno diferente, por lo cual, la información entregada debe ser adaptada a su realidad. Los pacientes son alumnos heterogéneos, por su edad (desde niños a ancianos), nivel cultural (algunos analfabetos), situación laboral (con estudiantes, dueñas de casa o profesionales) y pacientes con necesidades especiales (ciegos, minusválidos). La acción educativa debe considerar al paciente perteneciente a un entorno físico, involucrado en un grupo familiar y en una comunidad, el cual, influyen en su comportamiento y toma de decisiones frente a su salud ${ }^{6}$. Como se ha indicado, la educación del paciente necesita una pedagogía específica, basada en métodos centrados en el paciente que sufre una determinada afección y no en la enfermedad como entidad aislada del individuo ${ }^{4}$. Las actividades que se desarrollan en el marco de la educación a los pacientes dependen en gran medida de la buena voluntad de los profesionales como médicos y enfermeras, formados en disciplinas biomédicas. Estas actividades, por lo tanto, muchas veces se realizan sin un conocimiento pedagógico, sino por medio de la repetición de información. Por lo tanto, el profesional de salud debe contar con herramientas para provocar cambios cognitivos fundamentales para el éxito y adherencia al tratamiento que involucre ajustes y cambios desde rutina diaria a hábitos. Barrios et $\mathrm{al}^{7}$ señalan que este proceso educativo debe ser alineado con el contexto, los cambios socioculturales y las necesidades emergentes. Dadas estas necesidades, el estudio buscó comprender los significados que otorgan los profesionales de la salud a los procesos educativos que desarrollan en usuarios con insuficiencia renal crónica en un centro de diálisis.

\section{Material y Método}

El estudio fue cualitativo, de diseño fenomenológico e interpretativo. Se realizó en una unidad de diálisis en la que laboran profesionales médicos y profesionales de enfermería. Se utilizó la técnica de entrevista semiestructurada para la recogida de datos, siendo los participantes seleccionados a través del muestreo no probabilístico, por conveniencia. Los entrevistados fueron 3 médicos con conocimiento en patologías renales, con 3 años de experiencia, y 4 enfermeros con formación en diálisis, egresados desde hace tres años, hasta con 16 años de servicio.

Los criterios de inclusión utilizados fueron: participación voluntaria, profesionales de la salud médica y de enfermería y llevar tiempo de trabajo en la clínica diálisis mínimo de un año. 
Las entrevistas luego de ser grabadas, fueron transcritas en forma manual en formato texto para análisis, formando una serie de categorías emergentes y posteriormente desarrollando propiedades o subcategorías de las mismas, ofreciendo un marco descriptivo y explicativo.

Los participantes del estudio firmaron un consentimiento informado, garantizándose su confidencialidad y anonimato.

\section{Resultados}

Del análisis e interpretación de los datos cualitativos, proporcionada por los distintos participantes, se generaron 6 categorías presentes en la siguiente Tabla 1.
De acuerdo a los datos generados, las categorías se desarrollan de la siguiente forma. En razón de mantener el anonimato se denomina a los participantes de la investigación como El, equivalente a Entrevistado 1 y así sucesivamente:

\section{Categoría: Significado de Educación en Salud}

a) Subcategorías: Educación que inicia al proceso médico- enfermera

"significa... informar al paciente de los riesgos, de los cuidados, de la prevención del área de la salud y como afecta sistémicamente su patología, los síntomas que ellos puedan tener" E2.

b) Subcategorías: Educación que favorece la calidad de vida del paciente

"...es lo que le va a permitir al paciente empoderarse de su enfermedad y tomar cartas en el asunto, en forma cómo hacer... acciones claves como para su bienestar" E4.

\begin{tabular}{|c|c|c|}
\hline Nombre de la categoría & Definición & Subcategorías \\
\hline \multirow[t]{3}{*}{$\begin{array}{l}\text { Significado de Educación } \\
\text { en Salud. }\end{array}$} & \multirow{3}{*}{$\begin{array}{l}\text { Se comprende por educación en } \\
\text { salud a todo aquello que esté } \\
\text { relacionado con facilitar el proceso } \\
\text { de adaptación a la vida con la } \\
\text { enfermedad, lo cual transita entre } \\
\text { la entrega de información inicial } \\
\text { hasta pautas para un buen vivir en } \\
\text { conjunto. }\end{array}$} & $\begin{array}{l}\text { Educación que inicia al } \\
\text { proceso médico- enfermera. }\end{array}$ \\
\hline & & $\begin{array}{l}\text { Educación que favorece la } \\
\text { calidad de vida del paciente. }\end{array}$ \\
\hline & & $\begin{array}{l}\text { Educación que favorece la } \\
\text { calidad de vida de todos. }\end{array}$ \\
\hline \multirow{3}{*}{$\begin{array}{l}\text { Relevancia del proceso de } \\
\text { autocuidado. }\end{array}$} & \multirow{3}{*}{$\begin{array}{l}\text { Se refiere a la relevancia que } \\
\text { tiene el autocuidado para la vida } \\
\text { del paciente y porqué la educación } \\
\text { debe abordarla desde el inicio del } \\
\text { tratamiento. }\end{array}$} & Iniciación del autocuidado. \\
\hline & & Tránsito por la enfermedad. \\
\hline & & Otras prácticas relevantes. \\
\hline \multirow[t]{2}{*}{ Estrategias de Aprendizaje. } & \multirow{2}{*}{$\begin{array}{l}\text { Se refiere a la educación en salud } \\
\text { que requiere de estrategias para } \\
\text { generar aprendizaje, las cuales se } \\
\text { encuentran entre la entrega de } \\
\text { material y el diálogo. }\end{array}$} & Estrategias dialógicas. \\
\hline & & Materiales de Aprendizaje. \\
\hline \multirow[t]{2}{*}{$\begin{array}{l}\text { Variedades del uso del } \\
\text { material de apoyo. }\end{array}$} & \multirow{2}{*}{$\begin{array}{l}\text { Comprende al modo en que distintos } \\
\text { aspectos permiten o limitan usar } \\
\text { el material de apoyo oficial y } \\
\text { autogenerado por el centro en los } \\
\text { procesos educativos. }\end{array}$} & $\begin{array}{l}\text { Facilitadores del uso del } \\
\text { material de apoyo. }\end{array}$ \\
\hline & & $\begin{array}{l}\text { Obstaculizadores en el uso } \\
\text { del material. }\end{array}$ \\
\hline \multirow{3}{*}{$\begin{array}{l}\text { Facilitadores del proceso de } \\
\text { Enseñanza. }\end{array}$} & \multirow{6}{*}{$\begin{array}{l}\text { Se refiere a los distintos factores } \\
\text { que ayudan a los profesionales a } \\
\text { generar procesos de aprendizaje } \\
\text { efectivos y alcanzar los fines de la } \\
\text { educación en salud. } \\
\text { Corresponden a los distintos factores } \\
\text { que limitan a los profesionales de la } \\
\text { salud en el desarrollo del proceso de } \\
\text { aprendizaje. }\end{array}$} & Del paciente. \\
\hline & & Del profesional. \\
\hline & & Del entorno. \\
\hline \multirow{3}{*}{$\begin{array}{l}\text { Obstaculizadores del } \\
\text { proceso de Enseñanza. }\end{array}$} & & Del paciente. \\
\hline & & Del profesional. \\
\hline & & Del entorno. \\
\hline
\end{tabular}

Fuente: Elaboración Propia. c) Subcategorías: Educación que favorece la calidad de vida de todos "...métodos que se pueden aplicar de distintas formas hacia todo el equipo de salud tanto personal como del paciente para llevar a cabo un objetivo el común" E6.

En lo que respecta al significado de educación en salud para los profesionales, podemos señalar que los diferentes participantes lo relacionan con la educación al inicio del proceso, favoreciendo la calidad de vida tanto del paciente como de sus familias y entorno. Estos significados contemplan una diversidad de atribuciones como entregar información; integrar conocimientos; enseñar herramientas; empoderarse de su enfermedad para mejorar la adherencia y el autocuidado.

\section{Categoría: Relevancia del proceso de autocuidado}

a) Subcategorías: Iniciación del autocuidado

"el paciente en la etapa prediálisis es importante que reciba información básica sobre el tratamiento y 
el proceso de toma de decisiones, ya que preparar al paciente oportunamente para afrontar adecuadamente su enfermedad, puede evitar complicaciones y aminorar la ansiedad para la adaptación de la futura vida del paciente en la diálisis". E3.

b) Subcategorías: Tránsito por la enfermedad

"... porque diariamente cuando uno trata a los pacientes uno le va entregando educación sobre su alimentación, sobre su acceso vascular etc. sobre diferentes cosas que uno trata de enseñar" E7.

c) Subcategorías: Otras prácticas relevantes

"Para mejorar la calidad de vida de los pacientes, para que no.... Bueno solo ellos se van dando cuenta porque si no hacen casos lo que uno les dice lo que uno los educa terminan mal" E7.

El proceso de autocuidado del paciente es de relevancia para los profesionales desde el ingreso del paciente y durante su tratamiento, considerando refuerzo educativo diario para evitar complicaciones.

\section{Categoría: Estrategias de Aprendizaje}

a) Subcategorías: Estrategias dialógicas

"...yo trato de conversar con ellos en una forma más cercana, que ellos sientan un poco más de confianza con uno para poder contarles las cosas como un amigo que como un profesional a veces" El.

d) Subcategorías: Materiales de Aprendizaje

"... bueno más que nada hay trípticos con que se les refuerza a los pacientes, de acuerdo a la alimentación más que nada, pero generalmente todos los días verbal y recordar, recordar" E3.

Los profesionales de la salud utilizan como estrategias de aprendizaje principalmente la educación no formal a través del diálogo, generando confianza y reforzando la educación junto a la familia y a través de la experiencia, además de material de aprendizaje impreso para ayudar a evitar complicaciones y mejorar la adherencia al tratamiento.

Categoría: Variedades del uso del material de apoyo a) Subcategorías: Facilitadores del uso del material de apoyo

"... como explicarle didácticamente, con dibujos sirve bastante igual dentro de lo que se pueda no muy avanzado, pero bien básico eso ayuda igual interioriza mejor, como folletos, manuales de alimentación acá, mientras más didáctico sea.... Mejor" E4. b) Subcategorías: Obstaculizadores en el uso del material

".... un espacio para hacer educaciones que tengan una estructura preparada para con material audiovisual... no eso no se ve, más allá de los folletos de los que entrega la nutricionista el médico en momento de..." El.

Con respecto a las variedades del uso del material de apoyo, se observaron distintos aspectos que facilitan el uso como tiempo que los pacientes tienen durante el procedimiento, material impreso entregado por los profesionales que apoyan la educación y su refuerzo, o aspectos que limitan el uso de material de apoyo oficial y autogenerado por el centro en los procesos educativos, como la falta de espacio para realizar educación, material con términos poco coloquiales para el paciente y su contexto.

\section{Categoría: Facilitadores del proceso de Enseñanza}

a) Subcategorías: Del paciente

"... también la disposición de las personas, cuando la persona no tiene disposición a querer aprender o quieren que les enseñen y le da lo mismo también es una barrera importante" El.

b) Subcategorías: Del profesional

"La cercanía, la empatía con el tanto... del profesional con el paciente" E6.

c) Subcategorías: Del entorno

"Retroalimentaciones con ellos, porque a veces uno va y les habla y le habla y nunca les pregunta si entendieron o no, o a veces un error muy común es usar mucho lenguaje técnico" E5.

Existen diversos facilitadores del proceso de enseñanza relacionados con:

- El paciente: Su nivel educacional, su estado de salud, edad, confianza que presente con el profesional, disposición de aprender.

- El profesional: Interacción con el paciente, generar conocimiento con diálogo, experiencia, empatía, interacción con otros profesionales, generar confianza profesional-paciente, utilización de aspectos didácticos.

- El entorno: Generar conocimiento a través de material de apoyo, retroalimentación.

\section{Categoría: Obstaculizadores del proceso de Ense- ñanza}

a) Subcategorías: Del paciente

"Es netamente el tema del paciente, partiendo porque hay pacientes que tienen problemas auditivos, pacientes 
no videntes, que ya es una limitación física que complica, después los distintos niveles educativos" E4.

b) Subcategorías: Del profesional

"... se vuelve al final todo como una rutina y va cayendo en lo mismo, al menos que hay un interés como generalizado de educar que todos pongan de su parte, pero así no es difícil que nazca de uno" E7.

c) Subcategorías: Del entorno

"... yo creo que el tema es el medio de comunicación dificulta mucho en este tipo de pacientes y lo otro que obstaculiza son la familia, a veces la familia no son muy comunicativas con lo que les pasa a los pacientes en la casa, entonces igual a veces depende mucho de que tan preocupada son la familia" E2.

Como obstaculizadores tenemos relacionados con:

- El paciente: Presencia de patologías crónicas, bajo nivel socioeconómico, bajo nivel educacional, estado de salud, edad, falta de comprensión del paciente, limitaciones físicas del paciente.

- Del profesional: Frustración al educar paciente crónico, falta de motivación por el personal profesional, rutina, el cual, disminuye el interés en educación.

- Del entorno: Falta de tiempo para realizar educación al paciente, falta de comunicación con familiares, falta apoyo familiar, entorno del paciente, falta de espacio para realizar educación, distancia donde vive pacientes como limitación para educación.

\section{Discusión}

En este estudio al consultar a los profesionales sobre los significados que otorgaban a los procesos educativos de pacientes en diálisis, se generó una valiosa reflexión que abordó desde el ingreso del paciente, la entrega de información, conocimiento y herramientas para mejorar su autocuidado, además de la calidad de vida y cómo favorecer la esperanza de vida.

Un tema que adquirió particular relevancia fue el autocuidado, que para los profesionales se inicia en el momento del ingreso a diálisis y demanda un acompañamiento dedicado de los profesionales, porque es para ellos, la clave de un buen tratamiento. Según Tobón $^{8}$, se refiere a las prácticas cotidianas y las decisiones que realiza una persona y familia para cuidar de su salud. Son prácticas reconocidas como 'destrezas' empleadas por libre decisión, cuyo propósito es fortalecer o restablecer la salud y prevenir la enfermedad.
Este proceso ayuda a que los pacientes sean partícipes de su tratamiento en forma activa y logren resultados satisfactorios para ellos y sus familias, sin embargo los profesionales de la salud evidencian que este proceso demanda una permanente guía de parte de ellos, basada principalmente en su experiencia personal o trayectoria con paciente en diálisis.

Torres et al. ${ }^{9}$ señalan que parte de los autocuidados se relajan produciéndose hábitos distorsionados (aumento excesivo de peso, seguimiento incorrecto de la dieta, abandono de la adhesión al tratamiento...) No obstante, Santana et al..$^{10}$ consideran que hace falta información reiterada para los que olvidan, para los que por algún motivo no la tienen o para los que piensan que lo están haciendo bien y tienen algún aspecto mal aprendido.

De esta manera, la actividad permanente reconocida como valiosa por los profesionales del centro de diálisis, coincide con las recomendaciones teóricas y es además, relevante para que los profesionales se desempeñen adecuadamente.

Los elementos del estudio asociados a los significados para los profesionales fue la revelación de componentes didácticos con que se desarrollan los procesos educativos, los que se describen a continuación:

\section{a) Educación no formal}

La educación no formal está presente en diversos ámbitos del ser humano, en el área de la salud, Trilla ${ }^{11}$ señala que la actuación educativa constituye un elemento de primer orden en el incremento de la calidad de vida, la cual tiene como objeto el promover a la población en general una serie de conocimiento, hábitos y actitudes que inciden en la promoción de la salud. Barrios et al. ${ }^{7}$ considera elementos como las motivaciones, sentimientos, necesidades y los valores relacionados que hacen que el desempeño del profesional sea eficaz y eficiente dentro del contexto social en el cual se desarrollará.

De acuerdo con los autores, los profesionales de la salud operan fuera de la estructura de los sistemas de educación formal y generalmente libres de sus cánones y formalismos, resolviendo los intereses particulares y necesidades de aprendizaje de la población a la cual están dirigidas en su atención.

En el caso estudiado, para ello fue importante reforzar en forma continua dudas, especialmente cuando presentan complicaciones que pueden ser mejoradas a través de la educación, de modo de incentivar el autocuidado. 


\section{b) Diálogo}

Entre las estrategias de aprendizaje utilizadas por los profesionales de la salud se encuentran el diálogo, definida como un encuentro de seres humanos para encontrar un saber actuar común ${ }^{12}$. Esta actividad está arraigada en forma espontánea entre los profesionales y sus pacientes, según lo visto en este caso, lo cual es reforzado constantemente con un lenguaje tanto científico como coloquial, según su nivel educacional, edad y estado del paciente, apelando muchas veces a las emociones del paciente como estrategia, convirtiéndose en mediador de un proceso.

El diálogo es utilizado por los profesionales de la salud como una estrategia para entregar información en forma personalizada o grupal a personas con condiciones similares. Este tipo de estrategia se apropia de técnicas pedagógicas, muchas veces realizadas en forma autodidactas.

Surge la interrogante frente a este escenario, ¿cómo llegar a acuerdo, a la sincronización y la voluntad de diálogo? esto requiere voluntad desde ambas partes, empatizando, generando ambientes propicios que reconozcan por un lado la perdida el duelo y el proceso que este genera y por otro lado; generando asumir responsabilidad y adherencia al tratamiento asumiendo que el profesional lograra incorporar a sus quehaceres y habilidades un rol mediador, que frente a este realidad logre incorporar acuerdos y estrategias conjuntas para alcanzar un fin común.

\section{c) Experiencia}

Al igual que David Ausubel (1976) en su teoría del aprendizaje significativo, el aprendizaje experiencial plantea la necesidad de relacionar el contenido por aprender con las experiencias previas ${ }^{13}$.

La experiencia utilizada como método educativo es planteada por numerosos autores, cambiando la educación tradicional. Los profesionales de la salud no necesitan una sala de clases para realizar su educación, utilizan métodos menos convencionales como la experiencia personal y la trayectoria profesional, construyendo una relación mucha más cercana utilizando como vía principal para el ya mencionado diálogo.

Junto a lo anterior, se identificaron claramente factores que favorecen los procesos de enseñanza, dependiendo del:

- Paciente, disposición para aprender, confianza del paciente, edad, estado de salud, es decir, que no posea otras enfermedades asociadas y nivel educacional.
- Profesional; interacción con el paciente, diálogo, experiencia, empatía, generar confianza.

- Entorno; uso material de apoyo educativo, retroalimentación constante entre el profesional y el usuario.

Además, se identificaron factores que obstaculizan los procesos de enseñanza, dependiendo del:

- Paciente; presencia de patologías crónicas, bajo nivel socioeconómico y educacional, edad avanzada, estado de salud, limitaciones físicas del paciente, falta de comprensión del paciente.

- Profesional; frustración al educar por falta de interés o presencia constante de complicaciones, falta motivación, rutina laboral.

- Entorno, falta de comunicación con familiares, falta de tiempo para educar, entorno del paciente, falta de espacio para realizar educación, mayor apoyo del estado, distancia donde vive el paciente, cultura y familia.

En síntesis, queda en evidencia la importancia de los significados de la educación en salud para los profesionales, especialmente para comprender por qué realizan las prácticas educativas del modo en que lo hacen y potenciar con ello aspectos valiosos para mejorar la calidad de vida de los pacientes. Los profesionales de la salud trabajan en base a un método no formal, donde el diálogo y la experiencia o trayectoria resultan fundamentales para generar procesos de enseñanza.

Recibido: 20-11-2019

Revisado: 28-12-2019

Modificado: 27-01-2020

Aceptado: 12-06-2020

\section{Bibliografía}

1. Organización Mundial de la Salud. «WHO | Noncommunicable diseases», Organización Mundial de la Salud 2018. [Consultado 29 jul 2019]. Disponible en: https://www.who.int/en/news-room/factsheets/detail/noncommunicable-diseases. 
2. Alvarado L, Angulo D. Impacto del Rol de Enfermería en Pacientes con Insuficiencia Renal Crónica en el Instituto del Riñón y Diálisis Inrrsi San Martin Guayaquil. Tesis de grado; 2013. [consultado 17 jul 2019]. Disponible en: http://repositorio.ug.edu.ec/ bitstream/redug/8728/1/TESIS\%20FINAL.pdf

3. Guerra T, Díaz A, Vidal K. La educación como estrategia para mejorar la adherencia de los pacientes en terapia dialítica. Rev Cubana Enfermer 2010; 26(2):52-62.

4. Alfonso MT. Educación al Paciente Crónico. Rev Soc Esp Enferm Nefrol 1996;(1):6-15. [consultado 15 jul 2019]. Disponible en: https://www.revistaseden.org/files/art565_1.pdf.

5. Cristina Herzog 0. Educación al paciente y la familia en un programa de trasplante, experiencia en CLC. Rev Med Clin Condes 2010;21(2):293-9.

6. Rozo C. Fundamentos pedagógicos que sustentan el proceso de la educación en salud. Aquichan. 2002; 2(1):27-35.

7. Barrios Araya S, Masalán Apip MP, Cook MP. Educación en Salud: En la búsqueda de metodologías innovadoras. Cienc Enfermer 2011;17(1):57-69.
8. Tobón Correa 0 . El autocuidado una habilidad para vivir. Hacia promoc. Salud. 2015;8(1):38-50.

9. Torres J, Velasco P, Ila A. Intervención enseñanza a grupo en una unidad de hemodiálisis. Rev Soc Esp Enferm Nefrol. 2012;15(1):178-9.

10. Santana Díez MA, Aguirremota Corbera MR, Lodeiro Herraiz MP, González Vélez M, Castro Pereira S, Escudero Argaluza J. Resultados de un programa educativo con refuerzo audiovisual durante la sesión, en pacientes en hemodiálisis. Enfermer Nefrol 2017;20(2):126-31.

11. Trilla Bernet J. La educación fuera de la escuela. Ámbitos no formales y educación social. Barcelona: Ariel; 2003.

12. Leonello V, Oliveira M. Competencies for educational activities in nursing. Rev. Latino-Am. Enfermagem. 2008;16(2):177-83.

13. Díaz Barriga Arceo F. Cognición situada y estrategias para el aprendizaje significativo. REDIE 2003;5(2):105-17.

Este artículo se distribuye bajo una Licencia Creative Commons Atribución-NoComercial 4.0 Internacional. https://creativecommons.org/licenses/by-nc/4.0/ 